\title{
Accountability in International Disaster Response
}

\author{
Samuel J. Stratton, MD, MPH
}

It is a privilege to publish the 2011 Harvard Humanitarian Action Summit summaries in this issue of Prehospital and Disaster Medicine. The annual Humanitarian Action Summits, which began in 2009, have included international experts from all fields of the humanitarian community. The 2011 Summit was particularly important because those attending were able to review and analyze the humanitarian activities related to the 2010 Haiti Earthquake.

The Summit summaries address a range of humanitarian issues. They present data and thorough discussions that occurred during the Summit, with a broad overview of the developing practice, challenges, and technologies necessary for effective international disaster response. A most important theme throughout the summaries was the establishment of need for consensus on best practices in humanitarian actions and the need for accountability of those who act in a humanitarian capacity.

There is little doubt that the global humanitarian response to the Haiti Earthquake was important to the people of Haiti. While the international response to the Haiti earthquake was a remarkable example of the generosity of the global humanitarian community, there were occasional concerns regarding accountability for humanitarian actions that occurred during the Haiti response. The concept of humanitarian accountability is explained well in the Summit discussions of surgical procedures that occurred during the response to the Haiti Earthquake. In reporting data regarding Haiti Earthquake related amputations, it was reported that in some circumstances there was a lack of medical record keeping, lack of documented justification for amputations, laxity in obtaining informed consent for surgical procedures, and inconsistency in developing medical, mental health, and rehabilitation resources for some patients after amputations occurred. Most health practitioners providing similar medical care in the developed world would consider these reported problems to be less than acceptable. Yet, most of those who were health responders in Haiti were from developed world healthcare systems and this generates a paradox in that accepted health practices required at home may not have been adhered to in treating the Haiti disaster population.

Concern with humanitarian provider accountability for actions taken during the Haiti response was addressed honestly by those who attended the Humanitarian Summit. The Summit attendees are to be commended for scientific explorations of actions and procedures that can be embarrassing and politically sensitive to the humanitarian community. The Summit summaries do not cast blame or minimize the excellent work of the humanitarian community, but rather present objective assessments and data that will improve future humanitarian efforts throughout the globe. It is understood that providing health care to multiple victims in the austere settings of a major disaster is difficult and that the field environment in Haiti was far removed from the controlled environment of a modern hospital and health care delivery system. An excellent example of the clinical challenges faced in Haiti is illustrated in the summaries' discussion of the "surgeon's dilemma" or need for a surgeon faced with a severely crushed limb to weigh the benefit of early amputation to lessen loss of limb length and decrease risk of infection and necrosis versus an aggressive attempt to preserve the limb and accept the risk of systemic infection and metabolic derangement related to crush injury. This dilemma is much more difficult to deal with in a field hospital as opposed to a fully equipped developed world hospital. In essence, the clinical and health decisions required of humanitarian responders are difficult to compare to the same decisions that may be made in more ideal circumstances.

That said, most international humanitarian responders originate from developed countries and the standards of practice and expectations for patient care are established throughout the world. To relax those standards when responding to a population that 
has been made vulnerable because of a disaster is difficult to justify. The authors of the Summit summaries are to be commended for objectively addressing this issue of accountability in humanitarian response.

The Summit summaries also address the issue of integration of humanitarian response with a local health system. Throughout the summaries, it is acknowledged that as the humanitarian response wanes the local medical and social service system will quickly become the key element that provides for eventual success in maintaining and improving the health of a disaster stricken community. It is most often a bigger challenge in humanitarian response to bolster the local health system such that it can eventually provide for disaster victims than it is to mobilize and respond to an event.

Discussed in the summaries is the need for adjustment of humanitarian principles to address the on-going urbanization of populations throughout the world. Disaster science and humanitarian standards are in the early stages of addressing the challenges of developing urban environments. Dense urban areas with the risk of unstable building infrastructure, crowding, and mixing of cultures present a unique challenge for humanitarian programs. In addressing the challenges of the urban environment, the Summit summaries are cutting edge and provide enlightening information for all health providers.

Overall, the Summit summaries provide data and information that adds to the health and medical knowledge base. The objectivity in which difficult issues were addressed at the Summit is appreciated. In addition to helping develop consensus for humanitarian best practices and initiating the foundation for further developing accountability among humanitarian providers, the Humanitarian Action Summit summaries are an excellent source for future discussion and study in disaster health sciences. 